\title{
Traffic Network Equilibrium Problems with Capacity Constraints of Arcs and Linear Scalarization Methods
}

\author{
X. Q. Tian' ${ }^{1}$ and Y. D. $X u^{2}$ \\ ${ }^{1}$ Chongqing University of Science and Technology, Chongqing 401331, China \\ ${ }^{2}$ College of Mathematics and Statistics, Chongqing University, Chongqing 401331, China \\ Correspondence should be addressed to X. Q. Tian, tianxueq@126.com
}

Received 13 September 2012; Accepted 15 November 2012

Academic Editor: Nan-Jing Huang

Copyright (C) 2012 X. Q. Tian and Y. D. Xu. This is an open access article distributed under the Creative Commons Attribution License, which permits unrestricted use, distribution, and reproduction in any medium, provided the original work is properly cited.

Traffic network equilibrium problems with capacity constraints of arcs are studied. A (weak) vector equilibrium principle with vector-valued cost functions, which are different from the ones in the work of Lin (2010), and three kinds of parametric equilibrium flows are introduced. Some necessary and sufficient conditions for a (weak) vector equilibrium flow to be a parametric equilibrium flow are derived. Relationships between a parametric equilibrium flow and a solution of a scalar variational inequality problem are also discussed. Some examples are given to illustrate our results.

\section{Introduction}

The earliest traffic network equilibrium model was proposed by Wardrop [1] for a transportation network. After getting Wardrop's equilibrium principle, many scholars have studied variant kinds of network equilibrium models, see, for example, [2-5]. However, most of these equilibrium models are based on a single criterion. The assumption that the network users choose their paths based on a single criterion may not be reasonable. It is more reasonable to assume that no user will choose a path that incurs both a higher cost and a longer delay than some other paths. In other words, a vector equilibrium should be sought based on the principle that the flow of traffic along a path joining an $O-D$ pair is positive only if the vector cost of this path is the minimum possible among all the paths joining the same $O-D$ pair. Recently, equilibrium models based on multiple criteria or on a vector cost function have been proposed. In [6], Chen and Yen first introduced a vector equilibrium principle for vector traffic network without capacity constraints. In [7, 8], Khanh and Luu extended vector 
equilibrium principle to the case of capacity constraints of paths. For other results of vector equilibrium principle with capacity constraints of paths, we refer to [9-17].

Very recently, in $[18,19]$, Lin extended traffic network equilibrium principle to the case of capacity constraints of arcs and obtained a sufficient condition and stability results of vector traffic network equilibrium flows with capacity constraints of arcs. In [20], Xu et al. also considered that vector network equilibrium problems with capacity constraints of arcs. By virtue of a $\Delta$ function, which was introduced by Zaffaroni [21], the authors introduced a $\Delta$-equilibrium flow and a weak $\Delta$-equilibrium flow, respectively, and obtained sufficient and necessary conditions for a weak vector equilibrium flow to be a (weak) $\Delta$-equilibrium flow.

In this paper, our aim is to further investigate traffic network equilibrium problems with capacity constraints for arcs. We introduce a (weak) vector equilibrium principle with vector-valued cost functions, which are more reasonable from practical point of view than the ones in $[18,19]$. In order to obtain necessary and sufficient conditions for a (weak) vector equilibrium, we introduce three kinds of parametric equilibrium flows. Simultaneously, we also discuss relationships between a parametric equilibrium flow and a solution of a scalar variational inequality problem.

The outline of the paper is as follows. In Section 2, a (weak) equilibrium principle with capacity constraints of arcs is introduced. In Section 3, three kinds of parametric equilibrium flows are introduced. Some sufficient and necessary conditions for a (weak) vector equilibrium flow are obtained. Relationships between a parametric equilibrium flow and a solution of a scalar variational inequality problem are also discussed.

\section{Preliminaries}

For a traffic network, let $N$ and $E$ denote the set of nodes and directed arcs, respectively, and let $C=\left(c_{e}\right)_{e \in E}$ denote the capacity vector, where $c_{e}(>0)$ denotes the capacity of arc $e \in E$. Let $W$ denote the set of origin-destination $(O-D)$ pairs and let $D=\left(d_{w}\right)_{w \in W}$ denote the demand vector, where $d_{w}(>0)$ denotes the demand of traffic flow on $O-D$ pair $w$. A traffic network with capacity constraints of arcs is usually denoted by $G=(N, E, C, W, D)$. For each arc $e \in E$, the arc flow needs to satisfy the capacity constraints: $c_{e} \geq v_{e} \geq 0$, for each $e \in E$. For each $w \in W$, let $P_{w}$ denote the set of available paths joining $O-D$ pair $w$. Let $m=\sum_{w \in W}\left|P_{w}\right|$. For a given path $k \in P_{w}$, let $h_{k}$ denote the traffic flow on this path and $h=\left(h_{1}, h_{2}, \ldots, h_{m}\right) \in R^{m}$ is called a path flow. The path flow vector $h$ induces an arc flow $v_{e}$ on each $\operatorname{arc} e \in E$ given by

$$
v_{e}=\sum_{w \in W} \sum_{k \in P_{w}} \delta_{e k} h_{k}
$$

where $\delta_{e k}=1$ if the arc $e$ is contained in path $k$ and 0 , otherwise. Suppose that the demand of network flow is fixed for each $O-D$ pair $w$. We say that a path flow $h$ satisfies demand constraints

$$
\sum_{k \in P_{w}} h_{k}=d_{w}, \quad \forall w \in W
$$

A path flow $h$ satisfying the demand constraints and capacity constraints is called a feasible path flow. Let $H=\left\{h \in R_{+}^{m}\right.$ : for all $w \in W, \sum_{k \in P_{w}} h_{w}=d_{w}$ and for all $\left.e \in E, c_{e} \geq v_{e} \geq 0\right\}=$ $\left\{h \in R_{+}^{m}\right.$ : for all $w \in W, \sum_{k \in P_{w}} h_{w}=d_{w}$ and for all $\left.e \in E, c_{e} \geq \sum_{w \in W} \sum_{k \in P_{w}} \delta_{e k} h_{k} \geq 0\right\}$ and let $H \neq \emptyset$. Clearly, $H$ is convex and compact. Let $t_{e}\left(h_{k}\right): R_{+} \rightarrow R^{r}$ be a vector-valued cost 
function for the path $k$ on the arc $e$. Let $T_{k}(h): R_{+}^{m} \rightarrow R^{r}$ be a vector-valued cost function along the path $k$. Then the vector-valued cost on the path $k$ is equal to the sum of the all costs of the flow $h_{k}$ through arcs, which belong to the path $k$, that is,

$$
T_{k}(h)=\sum_{e \in E} \delta_{e k} t_{e}\left(h_{k}\right) .
$$

Let $T(h)=\left(T_{1}(h), T_{2}(h), \ldots, T_{m}(h)\right) \in R^{r \times m}$.

Remark 2.1. In $[18,19]$, Lin defined the vector cost function along the path $k$ as follows:

$$
\bar{T}_{k}(h)=\sum_{e \in E} \delta_{e k} \bar{t}_{e}(h)
$$

where $\bar{t}_{e}(h): R^{m} \rightarrow R^{r}$ be a vector-valued cost function for arc $e$. If the paths have common arcs, then the definition is unreasonable. The following example can illustrate the case.

Example 2.2. Consider the network problem depicted in Figure 1. $V=\{1,2,3,4\}, E=\left\{e_{1}, e_{2}\right.$, $\left.e_{3}, e_{4}, e_{5}\right\}, C=(3,2,2,4,3), W=\{(1,4),(3,4)\}, D=(3,4)$. The cost functions of arcs from $R$ to $R$ are, respectively, as follows:

$$
\begin{aligned}
& \bar{t}_{e_{1}}(h)=\bar{t}_{e_{1}}\left(v_{e_{1}}\right)=50 v_{e_{1}}+100, \quad \bar{t}_{e_{2}}(h)=\bar{t}_{e_{2}}\left(v_{e_{2}}\right)=20 v_{e_{2}}+500, \\
& \bar{t}_{e_{3}}(h)=\bar{t}_{e_{3}}\left(v_{e_{3}}\right)=60 v_{e_{3}}+100, \quad \bar{t}_{e_{4}}(h)=\bar{t}_{e_{4}}\left(v_{e_{4}}\right)=30 v_{e_{4}}+200, \\
& \bar{t}_{e_{5}}(h)=\bar{t}_{e_{5}}\left(v_{e_{5}}\right)=70 v_{e_{5}}+300 \text {. }
\end{aligned}
$$

For $O-D$ pair $(1,4): P_{(1,4)}$ includes path $1=(1,2,4)$ and path $2=(1,4)$, for $O-D$ pair $(3,4): P_{(3,4)}$ includes path $3=(3,2,4)$ and path $4=(3,4)$. And by $(2.4)$, we have

$$
\begin{array}{ll}
\bar{T}_{1}(h)=\bar{t}_{e_{1}}(h)+\bar{t}_{e_{5}}(h)=50 v_{e_{1}}+70 v_{e_{5}}+400, & \bar{T}_{2}(h)=\bar{t}_{e_{2}}(h)=20 v_{e_{2}}+500, \\
\bar{T}_{3}(h)=\bar{t}_{e_{3}}(h)+\bar{t}_{e_{5}}(h)=60 v_{e_{3}}+70 v_{e_{5}}+400, & \bar{T}_{4}(h)=\bar{t}_{e_{4}}(h)=30 v_{e_{4}}+200 .
\end{array}
$$

Then, for flow $h=\left(h_{1}, h_{2}, h_{3}, h_{4}\right)=(2,1,1,3)$, we have that arc flows

$$
v=\left(v_{e_{1}}, v_{e_{2}}, v_{e_{3}}, v_{e_{4}}, v_{e_{5}}\right)=(2,1,1,3,3) .
$$

It follows from (2.4) that

$$
\begin{aligned}
& \bar{T}_{1}(h)=\bar{t}_{e_{1}}\left(v_{e_{1}}\right)+\bar{t}_{e_{5}}\left(v_{e_{5}}\right)=50 \times 2+100+70 \times 3+300=710, \\
& \bar{T}_{3}(h)=\bar{t}_{e_{3}}\left(v_{e_{3}}\right)+\bar{t}_{e_{5}}\left(v_{e_{5}}\right)=60 \times 1+100+70 \times 3+300=670 .
\end{aligned}
$$

However, from the practical point of view, the cost values of the path 1 and path 3 with respect to $h$ are, respectively, as follows:

$$
\begin{aligned}
& T_{1}(h)=\bar{t}_{e_{1}}\left(h_{1}\right)+\bar{t}_{e_{5}}\left(h_{1}\right)=50 \times 2+100+70 \times 2+300=640, \\
& T_{3}(h)=\bar{t}_{e_{3}}\left(h_{3}\right)+\bar{t}_{e_{5}}\left(h_{3}\right)=60 \times 1+100+70 \times 1+300=530 .
\end{aligned}
$$

So, in this paper, we define the vector-valued cost function on a path as (2.3). 


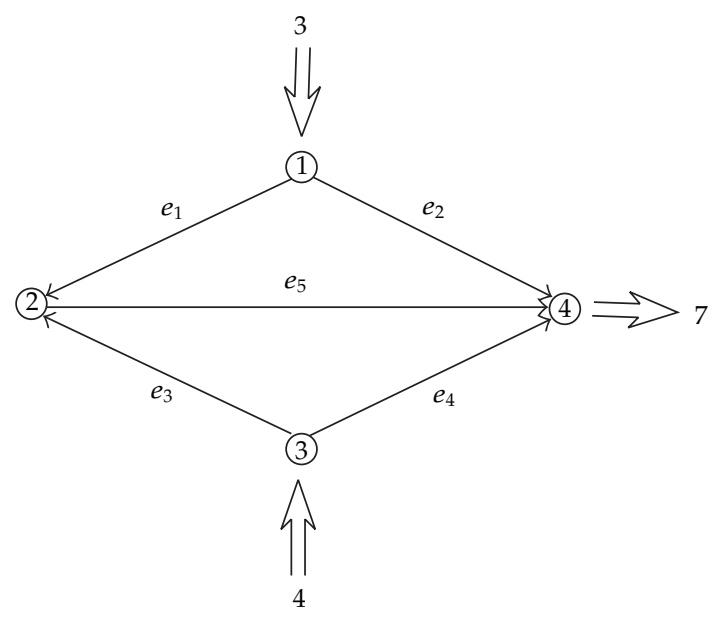

Figure 1: Network topology for an example.

In this paper, the cost space is an $r$-dimensional Euclidean space $R^{r}$, with the ordering cone $S=R_{+}^{r}$, a pointed, closed, and convex cone with nonempty interior int $S$. We define the ordering relation as follows:

$$
\begin{gathered}
x \leq s y, \quad \text { iff } y-x \in S ; \\
x<s y, \quad \text { iff } y-x \in \operatorname{int} S .
\end{gathered}
$$

The orderings $\geq_{S}$ and $>_{S}$ are defined similarly. In the sequel, we let the set $S^{+}:=\left\{\varphi \in R^{r}\right.$ : $\varphi(s) \geq 0$, for all $s \in S\}$ be the dual cone of $S$. Denote the interior of $S^{+}$by

$$
\operatorname{int} S^{+}:=\left\{\varphi \in R^{r}: \varphi(s)>0, \forall s \in S \backslash\{0\}\right\}
$$

Lemma 2.3 (see [22]). Consider

$$
\begin{aligned}
& S \backslash\{0\}:=\left\{x \in R^{r}: \varphi(x)>0, \forall \varphi \in \operatorname{int} S^{+}\right\}, \\
& \text {int } S:=\left\{x \in R^{r}: \varphi(x)>0, \forall \varphi \in S^{+} \backslash\{0\}\right\} .
\end{aligned}
$$

Definition 2.4 (see $[18,19])$. Assume that a flow $h \in H$,

(i) for $e \in E$, if $v_{e}=c_{e}$, then arc $e$ is said to be a saturated arc of flow $h$, otherwise a nonsaturated arc of flow $h$.

(ii) for $k \in \bigcup_{w \in W} P_{w}$, if there exists a saturated arc $e$ of flow $h$ such that $e$ belongs to path $k$, then path $k$ is said to be a saturated path of flow $h$, otherwise a nonsaturated path of flow $h$.

We introduced the following vector equilibrium principle and weak vector equilibrium principle. 
Definition 2.5 (vector equilibrium principle). A flow $h \in H$ is said to be a vector equilibrium flow if for all $w \in W$, for all $k, j \in P_{w}$, we have

$$
T_{k}(h)-T_{j}(h) \in S \backslash\{0\} \Longrightarrow h_{k}=0 \text { or path } j \text { is a saturated path of flow } h \text {. }
$$

Definition 2.6 (weak vector equilibrium principle). A flow $h \in H$ is said to be a weak vector equilibrium flow if for all $w \in W$, for all $k, j \in P_{w}$, we have

$$
T_{k}(h)-T_{j}(h) \in \operatorname{int} S \Longrightarrow h_{k}=0 \text { or path } j \text { is a saturated path of flow } h \text {. }
$$

If for all $e \in E, c_{e}=c \geq \sum_{w \in W} d_{w}$, then the capacity constraints of arcs are invalid, in this case, the traffic equilibrium problem with capacity constraints of arcs reduces to the traffic equilibrium problem without capacity constraints of arcs.

\section{Sufficient and Necessary Conditions for a (Weak) Vector Equilibrium Flow}

In this section, we introduce an int $S^{+}$-parametric equilibrium flow, a $S^{+} \backslash\{0\}$-parametric equilibrium flow and a $\varphi$-parametric equilibrium flow, respectively. By using the three new concepts, we can obtain some sufficient and necessary conditions of a vector equilibrium flow and a weak vector equilibrium flow, respectively.

Definition 3.1. A flow $h \in H$ is said to be in int $S^{+}$-parametric equilibrium if for all $w \in W$, for all $k, j \in P_{w}$ and for all $\varphi \in$ int $S^{+}$, we have

$$
\varphi\left(T_{k}(h)-T_{j}(h)\right)>0 \Longrightarrow h_{k}=0 \text { or path } j \text { is a saturated path of flow } h \text {. }
$$

Definition 3.2. A flow $h \in H$ is said to be in $S^{+} \backslash\{0\}$-parametric equilibrium if for all $w \in W$, for all $k, j \in P_{w}$ and for all $\varphi \in S^{+} \backslash\{0\}$, we have

$$
\varphi\left(T_{k}(h)-T_{j}(h)\right)>0 \Longrightarrow h_{k}=0 \text { or path } j \text { is a saturated path of flow } h \text {. }
$$

Definition 3.3. Let a $\varphi \in S^{+} \backslash\{0\}$ be given. A flow $h \in H$ is said to be in $\varphi$-parametric equilibrium flow if for all $w \in W$ and for all $k, j \in P_{w}$, we have

$$
\varphi\left(T_{k}(h)-T_{j}(h)\right)>0 \Longrightarrow h_{k}=0 \text { or path } j \text { is a saturated path of flow } h \text {. }
$$

The int $S^{+}$-equilibrium flow and $\varphi$-parametric equilibrium flow for some $\varphi \in \operatorname{int} S^{+}$ are defined in Definitions 3.1 and 3.2, respectively. They can be used to characterize vector equilibrium flow in the following theorems.

Theorem 3.4. A flow $h \in H$ is in vector equilibrium if and only if the flow $h$ is in int $S^{+}$-parametric equilibrium.

Proof. It can get immediately the above conclusion by Lemma 2.3. Thus the proof is omitted here. 
Theorem 3.5. If there exists $\varphi \in$ int $S^{+}$such that a flow $h \in H$ is in $\varphi$-parametric equilibrium, then the flow $h$ is in vector equilibrium.

Proof. Suppose that for any $O-D$ pair $w \in W$, for all $k, j \in P_{w}$, we have

$$
T_{k}(h)-T_{j}(h) \in S \backslash\{0\} .
$$

By $\varphi \in \operatorname{int} S^{+}$and Lemma 2.3, we get immediately

$$
\varphi\left[T_{k}(h)-T_{j}(h)\right]>0
$$

Since $h$ is in $\varphi$-parametric equilibrium, we have

$$
h_{k}=0 \text { or path } j \text { is a saturated path of flow } h \text {. }
$$

Thus, the flow $h \in H$ is in vector equilibrium.

Now, we give the following example to illustrate Theorem 3.5.

Example 3.6. Consider the network problem depicted in Figure 2. $N=\{1,2,3,4\}, E=\left\{e_{1}, e_{2}\right.$, $\left.e_{3}, e_{4}, e_{5}, e_{6}\right\}, C=(3,3,3,2,3,4)^{T}, W=\{(1,4),(3,4)\}, D=(6,4)$. The cost functions of arcs from $R$ to $R^{2}$ are defined as follows:

$$
\begin{gathered}
t_{e_{1}}\left(h_{1}\right)=\left(\begin{array}{c}
h_{1}^{2}+1 \\
2 h_{1}
\end{array}\right), \quad t_{e_{2}}\left(h_{2}\right)=\left(\begin{array}{c}
5 h_{2} \\
3 h_{2}^{2}
\end{array}\right), \quad t_{e_{3}}\left(h_{3}\right)=\left(\begin{array}{c}
h_{3}^{2}+7 \\
5 h_{3}
\end{array}\right), \\
t_{e_{4}}\left(h_{4}\right)=\left(\begin{array}{c}
2 h_{4}+1 \\
3 h_{4}
\end{array}\right), \quad t_{e_{5}}\left(h_{5}\right)=\left(\begin{array}{c}
3 h_{5}^{2} \\
6 h_{5}
\end{array}\right), \quad t_{e_{6}}\left(h_{1}\right)=\left(\begin{array}{c}
h_{1}^{2} \\
2 h_{1}
\end{array}\right), \quad t_{e_{6}}\left(h_{4}\right)=\left(\begin{array}{c}
h_{4}^{2} \\
2 h_{4}
\end{array}\right) .
\end{gathered}
$$

Then, we have

$$
\begin{gathered}
T_{1}(h)=t_{e_{1}}\left(h_{1}\right)+t_{e_{6}}\left(h_{1}\right)=\left(\begin{array}{c}
2 h_{1}^{2}+1 \\
4 h_{1}
\end{array}\right), \quad T_{4}(h)=t_{e_{4}}\left(h_{4}\right)+t_{e_{6}}\left(h_{4}\right)=\left(\begin{array}{c}
h_{4}^{2}+2 h_{4}+1 \\
5 h_{4}
\end{array}\right), \\
T_{2}(h)=t_{e_{2}}\left(h_{2}\right)=\left(\begin{array}{c}
5 h_{2} \\
3 h_{2}^{2}
\end{array}\right), \quad T_{3}(h)=t_{e_{3}}\left(h_{3}\right)=\left(\begin{array}{c}
h_{3}^{2}+7 \\
5 h_{3}
\end{array}\right), \quad T_{5}(h)=t_{e_{5}}\left(h_{5}\right)=\left(\begin{array}{c}
3 h_{5}^{2} \\
6 h_{5}
\end{array}\right) .
\end{gathered}
$$

Taking $h^{*}=(2,2,2,2,2)^{\prime} \in H$, then there exists $\bar{\varphi}=(1,1) \in \operatorname{int} R_{+}^{2}$ such that the flow $h^{*}$ is in $\bar{\varphi}$-parametric equilibrium. Thus, by Theorem 3.5, we have that the flow $h^{*}$ is in vector equilibrium.

For weak vector equilibrium flows, we have following similar results.

Theorem 3.7. A path flow $h \in H$ is in weak vector equilibrium if and only if the flow $h$ is in $S^{+} \backslash\{0\}-$ parametric equilibrium. 


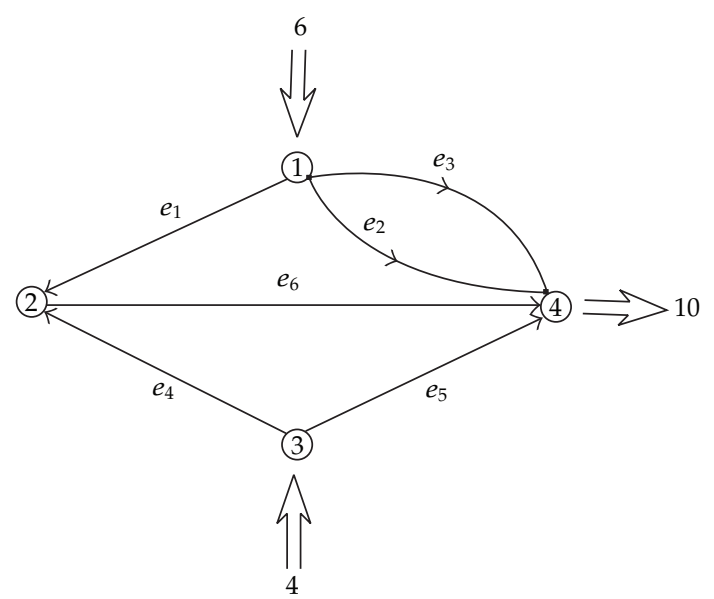

Figure 2: Network topology for an example.

Theorem 3.8. If there exists $\varphi \in S^{+} \backslash\{0\}$ such that a path flow $h \in H$ is in $\varphi$-parametric equilibrium, then the flow $h$ is in weak vector equilibrium.

From Theorems 3.4-3.8, we can get immediately the following corollaries.

Corollary 3.9. If there exists $\varphi \in$ int $S^{+}$such that a flow $h \in H$ is in $\varphi$-parametric equilibrium, then the flow $h$ is in int $S^{+}$-parametric equilibrium.

Corollary 3.10. If there exists $\varphi \in S^{+} \backslash\{0\}$ such that a flow $h \in H$ is in $\varphi$-parametric equilibrium, then the flow $h$ is in $S^{+} \backslash\{0\}$-parametric equilibrium.

Remark 3.11. When a flow $h \in H$ is in int $S^{+}$-parametric equilibrium, then, the flow $h$ may not be in $\varphi$-parametric equilibrium for some $\varphi \in \operatorname{int} S^{+}$. Of course, when a flow $h \in H$ is in $S^{+} \backslash\{0\}$-parametric equilibrium, then, the flow $h$ may not be in $\varphi$-parametric equilibrium for some $\varphi \in S^{+} \backslash\{0\}$. The following example can explain these cases.

Example 3.12. Consider the network problem depicted in Figure 1. $N=\{1,2,3,4\}, E=$ $\left\{e_{1}, e_{2}, e_{3}, e_{4}, e_{5},\right\}, C=(3,3,2,4,3),, W=\{\{1,4\},\{3,4\}\}, D=\{3,4\}$. Let the cost functions of arcs are defined as follows:

$$
\begin{gathered}
t_{e_{1}}\left(h_{1}\right)=\left(\begin{array}{c}
h_{1}^{2}+2 \\
h_{1}^{2}+3
\end{array}\right), \quad t_{e_{2}}\left(h_{2}\right)=\left(\begin{array}{c}
h_{2}^{2}+h_{2}+2 \\
h_{2}+2
\end{array}\right), \quad t_{e_{3}}\left(h_{3}\right)=\left(\begin{array}{c}
3 h_{3}^{2}+2 \\
2 h_{3}+2
\end{array}\right), \\
t_{e_{4}}\left(h_{4}\right)=\left(\begin{array}{c}
2 h_{4}+4 \\
h_{4}+1
\end{array}\right), \quad t_{e_{5}}\left(h_{1}\right)=\left(\begin{array}{c}
h_{1}^{2}+2 \\
2 h_{1}
\end{array}\right), \quad t_{e_{5}}\left(h_{3}\right)=\left(\begin{array}{c}
h_{3}^{2}+2 \\
2 h_{3}
\end{array}\right) .
\end{gathered}
$$

Then, we have

$$
\begin{aligned}
& T_{1}(h)=t_{e_{1}}\left(h_{1}\right)+t_{e_{6}}\left(h_{1}\right)=\left(\begin{array}{c}
2 h_{1}^{2}+4 \\
h_{1}^{2}+2 h_{1}+3
\end{array}\right), \quad T_{2}(h)=t_{e_{2}}\left(h_{2}\right)=\left(\begin{array}{c}
h_{2}^{2}+h_{2}+2 \\
h_{2}+2
\end{array}\right), \\
& T_{3}(h)=t_{e_{3}}\left(h_{3}\right)=t_{e_{3}}\left(h_{3}\right)+t_{e_{5}}\left(h_{3}\right)=\left(\begin{array}{c}
4 h_{3}^{2}+4 \\
4 h_{3}+2
\end{array}\right), \quad T_{4}(h)=t_{e_{4}}\left(h_{4}\right)=\left(\begin{array}{c}
2 h_{4}+4 \\
h_{4}+1
\end{array}\right) .
\end{aligned}
$$


Taking

$$
h^{*}=(1,2,1,3)^{\prime},
$$

we have

$$
T_{1}\left(h^{*}\right)=\left(\begin{array}{l}
6 \\
6
\end{array}\right), \quad T_{2}\left(h^{*}\right)=\left(\begin{array}{l}
8 \\
4
\end{array}\right), \quad T_{3}\left(h^{*}\right)=\left(\begin{array}{l}
8 \\
6
\end{array}\right), \quad T_{4}\left(h^{*}\right)=\left(\begin{array}{c}
10 \\
4
\end{array}\right) .
$$

Thus, by Definitions 3.1 and 3.2, we know that the flow $h^{*}$ is a int $S^{+}$-parametric equilibrium flow and is a $S^{+} \backslash\{0\}$-parametric equilibrium flow as well. On the other hand, for $\varphi=$ $(1,1 / 2)^{\prime} \in \operatorname{int} S^{+} \subset S^{+} \backslash\{0\}$, there exists $w=\{1,4\}$ and path $1,2 \in P_{w}$, we have

$$
\varphi\left[T_{2}\left(h^{*}\right)-T_{1}\left(h^{*}\right)\right]=1>0 .
$$

But, $h_{2}=2>0$ and path 1 is nonsaturated path of $h^{*}$. Thus, it follows from Definition 3.3 that the flow $h^{*}$ is not in $\varphi$-parametric equilibrium.

Theorem 3.13. Let $\varphi \in S^{+} \backslash\{0\}$ be given. A flow $h \in H$ is in $\varphi$-parametric equilibrium if the flow $h$ solves the following scalar variational inequality:

$$
\sum_{w \in W} \sum_{p \in P_{w}} \varphi\left(T_{p}(h)\right)\left(f_{p}-h_{p}\right) \geq 0, \quad \forall f \in H .
$$

Proof. Assume that $h \in H$ solves above scalar variational inequality problem. For all $w \in W$, for all $k, j \in P_{w}$, if $\varphi\left[T_{k}(h)-T_{j}(h)\right]=\varphi\left[T_{k}(h)\right]-\varphi\left[T_{j}(h)\right]>0$ and path $j$ is nonsaturated path of flow $h$, we need to prove that $h_{k}=0$. Denote that $p_{j}=\{e \in E \mid \operatorname{arc} e$ belongs to path $j\}$. If the conclusion is false, then

$$
\epsilon=\min \left\{\min _{e \in p_{j}}\left(c_{e}-v_{e}\right), h_{k}\right\}>0
$$

Construct a flow $f$ as follows:

$$
f=\left(f_{l}\right)= \begin{cases}h_{l}, & \text { if } l \neq k \text { or } j, \\ \left(h_{k}-\epsilon\right), & \text { if } l=k, \\ \left(h_{j}+\epsilon\right), & \text { if } l=j .\end{cases}
$$

It is easy to verify that

$$
f \in H \text {. }
$$


It follows readily that

$$
\begin{aligned}
\sum_{w \in W} \sum_{p \in P_{w}} \varphi\left(T_{p}(h)\right)\left(f_{p}-h_{p}\right) & =\varphi\left(T_{k}(h)\right)\left(f_{k}-h_{k}\right)+\varphi\left(T_{j}(h)\right)\left(f_{j}-h_{j}\right) \\
& =\epsilon\left(\varphi\left[T_{j}(h)\right]-\varphi\left[T_{k}(h)\right]\right) \\
& <0,
\end{aligned}
$$

which contradicts (3.14). Thus, $h$ is in $\varphi$-parametric equilibrium and the proof is complete.

From Theorems 3.4-3.13, we can get the following corollary.

Corollary 3.14. If there exists $\varphi \in \operatorname{int} S^{+}\left(\varphi \in S^{+} \backslash\{0\}\right)$ such that a flow $h \in H$ is a solution of the following scalar variational inequality:

$$
\sum_{w \in W} \sum_{p \in P_{w}} \varphi\left(T_{p}(h)\right)\left(f_{p}-h_{p}\right) \geq 0, \quad \forall f \in H,
$$

then the flow $h$ is in (weak) vector equilibrium.

Remark 3.15. We can prove that the the converse of Theorem 3.13 is valid when the traffic network equilibrium problem without capacity constraints of arcs, such as traffic network equilibrium problems without capacity constraints or with capacity constraints of paths. The result will be showed on Theorem 3.18. But, if the traffic network equilibrium problem with capacity constraints of arcs, then the converse of Theorem 3.13 may not hold. The following example is given to illustrate the case.

Example 3.16. Consider the network problem depicted in Figure 1. $N=\{1,2,3,4\}, E=\left\{e_{1}, e_{2}\right.$, $\left.e_{3}, e_{4}, e_{5}\right\}, C=(3,2,2,4,3), W=\{\{1,4\},\{3,4\}\}, D=\{3,4\}$. Let the cost functions of arcs are defined as follows:

$$
\begin{gathered}
t_{e_{1}}\left(h_{1}\right)=\left(\begin{array}{l}
h_{1} \\
h_{1}^{2}
\end{array}\right), \quad t_{e_{2}}\left(h_{2}\right)=\left(\begin{array}{c}
h_{2}^{2}+3 h_{2}+5 \\
h_{2}^{3}+4 h_{2}+3
\end{array}\right), \quad t_{e_{3}}\left(h_{3}\right)=\left(\begin{array}{c}
h_{3}^{3}+3 \\
h_{3}^{2}+4
\end{array}\right), \\
t_{e_{4}}\left(h_{4}\right)=\left(\begin{array}{c}
h_{4}+4 \\
h_{4}+4
\end{array}\right), \quad t_{e_{5}}\left(h_{1}\right)=\left(\begin{array}{c}
h_{1}^{2}+1 \\
h_{1}
\end{array}\right), \quad t_{e_{5}}\left(h_{3}\right)=\left(\begin{array}{c}
h_{3}^{2}+1 \\
h_{3}
\end{array}\right) .
\end{gathered}
$$

Then, we have

$$
\begin{gathered}
T_{1}(h)=t_{e_{1}}\left(h_{1}\right)+t_{e_{5}}\left(h_{1}\right)=\left(\begin{array}{c}
h_{1}^{2}+h_{1}+1 \\
h_{1}^{2}+h_{1}
\end{array}\right), \quad T_{2}(h)=t_{e_{2}}\left(h_{2}\right)=\left(\begin{array}{c}
h_{2}^{2}+3 h_{2}+5 \\
h_{2}^{3}+4 h_{2}+3
\end{array}\right), \\
T_{3}(h)=t_{e_{3}}\left(h_{3}\right)+t_{e_{5}}\left(h_{3}\right)=\left(\begin{array}{c}
h_{3}^{3}+h_{3}^{2}+4 \\
h_{3}^{2}+h_{3}+4
\end{array}\right), \quad T_{4}(h)=t_{e_{4}}\left(h_{4}\right)=\left(\begin{array}{c}
h_{4}+4 \\
h_{4}+4
\end{array}\right) .
\end{gathered}
$$


Taking

$$
h^{*}=(2,1,1,3)^{\prime},
$$

we have

$$
T_{1}\left(h^{*}\right)=\left(\begin{array}{l}
7 \\
6
\end{array}\right), \quad T_{2}\left(h^{*}\right)=\left(\begin{array}{l}
9 \\
8
\end{array}\right), \quad T_{3}\left(h^{*}\right)=\left(\begin{array}{l}
6 \\
6
\end{array}\right), \quad T_{4}\left(h^{*}\right)=\left(\begin{array}{l}
7 \\
7
\end{array}\right)
$$

Then for any $\varphi \in \operatorname{int} S^{+}\left(\varphi \in S^{+} \backslash\{0\}\right)$, we have

$$
\begin{aligned}
& \varphi\left[T_{2}\left(h^{*}\right)-T_{1}\left(h^{*}\right)\right]>0, \\
& \varphi\left[T_{4}\left(h^{*}\right)-T_{3}\left(h^{*}\right)\right]>0,
\end{aligned}
$$

and path 1 is a saturated arc path of $h^{*}$, and path 3 is a saturated arc path of $h^{*}$ as well. Thus, the flow $h^{*}$ is a $\varphi$-parametric equilibrium flow by Definition 3.3. However, taking $f=$ $(3,0,0,4)^{\prime} \in H$, we have

$$
\sum_{w \in W} \sum_{p \in P_{w}} T_{p}\left(h^{*}\right)\left(f_{p}-h_{p}^{*}\right)=(-1,-1)^{\prime} .
$$

Thus, for any $\varphi \in \operatorname{int} S^{+}\left(\varphi \in S^{+} \backslash\{0\}\right)$, we can always get

$$
\sum_{w \in W} \sum_{p \in P_{w}} \varphi\left(T_{p}\left(h^{*}\right)\right)\left(f_{p}-h_{p}^{*}\right)<0
$$

Therefore, the converse of Theorem 3.13 is not valid.

The following theorem shows that the converse of Theorem 3.13 is valid when the traffic equilibrium problem with capacity constraints of paths. The proof is similar when the traffic network equilibrium problem without capacity constraints. Let

$$
K:=\left\{h \mid \lambda \leq h \leq \mu, \sum_{p \in P_{w}} h_{p}=d_{w}, \forall w \in W\right\},
$$

be the feasible set of traffic network equilibrium problem with capacity constraints of paths, where $\lambda=\left(\lambda_{1}, \lambda_{2}, \ldots, \lambda_{m}\right)$ and $\mu=\left(\mu_{1}, \mu_{2}, \ldots, \mu_{m}\right)$ are lower and upper capacity constraints of paths, respectively. The $\varphi$-parametric equilibrium principle of traffic equilibrium problem with capacity constraints of paths is as follows.

Definition 3.17. Let a $\varphi \in S^{+} \backslash\{0\}$ be given. A flow $h \in H$ is said to be in $\varphi$-parametric equilibrium flow if for all $w \in W$ and for all $k, j \in P_{w}$, we have

$$
\varphi\left(T_{k}(h)-T_{j}(h)\right)>0 \Longrightarrow h_{j}=\mu_{j} \text { or } h_{k}=\lambda_{k}
$$


Theorem 3.18. Let $\varphi \in S^{+} \backslash\{0\}$ be given. A path $h \in K$ is in $\varphi$-parametric equilibrium if and only if the flow $h$ solves the following scalar variational inequality:

$$
\sum_{w \in W} \sum_{p \in P_{w}} \varphi\left(T_{p}(h)\right)\left(f_{p}-h_{p}\right) \geq 0, \quad \forall f \in K .
$$

Proof. From Theorem 3.13, we only prove necessity. So, we set

$$
A_{w}:=\left\{v \in P_{w} \mid h_{v}>\lambda_{v}\right\}, \quad B_{w}:=\left\{u \in P_{w} \mid h_{u}<\mu_{u}\right\} .
$$

It follows from the definition of the $\varphi$-parametric equilibrium flow that

$$
\varphi\left[T_{u}(h)\right] \geq \varphi\left[T_{v}(h)\right], \quad \forall u \in B_{w}, v \in A_{w} .
$$

Thus, there exists a $\gamma_{w} \in R$ such that

$$
\min _{u \in B_{w}} \varphi\left[T_{u}(h)\right] \geq \gamma_{w} \geq \max _{v \in A_{w}} \varphi\left[T_{v}(h)\right]
$$

Let $f \in K$ be arbitrary. Then, for every $r \in P_{w}$, we consider three cases.

Case 1. If $\varphi\left[T_{r}(h)\right]<\gamma_{w}$, then $r \notin B_{w}$. Hence, $h_{r}=\mu_{r}, f_{r}-h_{r} \leq 0$ and

$$
\left[\varphi\left(T_{k}(h)\right)-\gamma_{w}\right]\left(f_{r}-h_{r}\right) \geq 0
$$

Case 2. If $\varphi\left[T_{r}(h)\right]>\gamma_{w}$, then $r \notin A_{w}$. Hence, $h_{r}=\lambda_{r}, f_{r}-h_{r} \geq 0$ and

$$
\left[\varphi\left(T_{k}(h)\right)-\gamma_{w}\right]\left(f_{r}-h_{r}\right) \geq 0
$$

Case 3. If $\varphi\left[T_{r}(h)\right]=\gamma_{w}$, then we have

$$
\left[\varphi\left(T_{k}(h)\right)-\gamma_{w}\right]\left(f_{r}-h_{r}\right) \geq 0
$$

From (3.33), (3.34), and (3.35), we have

$$
\sum_{w \in W} \sum_{p \in P_{w}} \varphi\left(T_{p}(h)\right)\left(f_{p}-h_{p}\right) \geq \sum_{w \in W} \sum_{p \in P_{w}} \gamma_{w}\left(d_{w}-d_{w}\right)=0 .
$$

Thus, the proof is complete.

\section{Conclusions}

In this paper, we have studied traffic network equilibrium problems with capacity constraints of arcs. We have introduced some new parametric equilibrium flows, such as: $S^{+} \backslash\{0\}$-parametric equilibrium flows, int $S^{+}$-parametric equilibrium flows, and $\varphi$-parametric equilibrium 
flows. By using these new concepts, we have characterized vector equilibrium problems on networks and derived some necessary and sufficient conditions for a (weak) vector equilibrium flow.

\section{References}

[1] J. Wardrop, "Some theoretical aspects of road traffic research," Proceedings of the Institute of Civil Engineers, vol. 1, pp. 325-378, 1952.

[2] Q. Meng, Y. K. Huang, and R. L. Cheu, "A note on supply chain network equilibrium models," Transportation Research E, vol. 43, no. 1, pp. 60-71, 2007.

[3] A. Nagurney and J. Dong, "A multiclass, multicriteria traffic network equilibrium model with elastic demand," Transportation Research B, vol. 36, no. 5, pp. 445-469, 2002.

[4] A. Nagurney, J. Dong, and D. Zhang, "A supply chain network equilibrium model," Transportation Research E, vol. 38, no. 5, pp. 281-303, 2002.

[5] A. Nagurney, "On the relationship between supply chain and transportation network equilibria: a supernetwork equivalence with computations," Transportation Research E, vol. 42, no. 4, pp. 293-316, 2006.

[6] G. Y. Chen and N. D. Yen, On the Variational Inequality Model For Network Equilibrium, vol. 724, Department of Mathematics, University of Pisa, 1993, Internal Report 3.

[7] P. Q. Khanh and L. M. Luu, "On the existence of solutions to vector quasivariational inequalities and quasicomplementarity problems with applications to traffic network equilibria," Journal of Optimization Theory and Applications, vol. 123, no. 3, pp. 533-548, 2004.

[8] P. Q. Khanh and L. M. Luu, "Some existence results for vector quasivariational inequalities involving multifunctions and applications to traffic equilibrium problems," Journal of Global Optimization, vol. 32, no. 4, pp. 551-568, 2005.

[9] G. Y. Chen, C. J. Goh, and X. Q. Yang, "Vector network equilibrium problems and nonlinear scalarization methods," Mathematical Methods of Operations Research, vol. 49, no. 2, pp. 239-253, 1999.

[10] T. C. E. Cheng and Y. N. Wu, "A multiproduct, multicriterion supply-demand network equilibrium model," Operations Research, vol. 54, no. 3, pp. 544-554, 2006.

[11] S. J. Li, X. Q. Yang, and G. Y. Chen, "A note on vector network equilibrium principles," Mathematical Methods of Operations Research, vol. 64, no. 2, pp. 327-334, 2006.

[12] S. J. Li, K. L. Teo, and X. Q. Yang, "A remark on a standard and linear vector network equilibrium problem with capacity constraints," European Journal of Operational Research, vol. 184, no. 1, pp. 13-23, 2008.

[13] S. J. Li, K. L. Teo, and X. Q. Yang, "Vector equilibrium problems with elastic demands and capacity constraints," Journal of Global Optimization, vol. 37, no. 4, pp. 647-660, 2007.

[14] C. J. Goh and X. Q. Yang, "Vector equilibrium problem and vector optimization," European Journal of Operational Research, vol. 116, no. 3, pp. 615-628, 1999.

[15] F. Raciti, "Equilibrium conditions and vector variational inequalities: a complex relation," Journal of Global Optimization, vol. 40, no. 1-3, pp. 353-360, 2008.

[16] X. Q. Yang and C. J. Goh, “On vector variational inequalities: application to vector equilibria," Journal of Optimization Theory and Applications, vol. 95, no. 2, pp. 431-443, 1997.

[17] D. T. Luc, M. Rocca, and M. Papalia, "Equilibrium in a vector supply-demand network with capacity constraints," Applicable Analysis, vol. 90, no. 6, pp. 1029-1045, 2011.

[18] Z. Lin, "The study of traffic equilibrium problems with capacity constraints of arcs," Nonlinear Analysis. Real World Applications, vol. 11, no. 4, pp. 2280-2284, 2010.

[19] Z. Lin, "On existence of vector equilibrium flows with capacity constraints of arcs," Nonlinear Analysis. Theory, Methods E Applications A, vol. 72, no. 3-4, pp. 2076-2079, 2010.

[20] Y. D. Xu, S. J. Li, and K. L. Teo, "Vector network equilibrium problems with capacity constraints of arcs," Transportation Research E, vol. 48, pp. 567-577, 2012.

[21] A. Zaffaroni, "Degrees of efficiency and degrees of minimality," SIAM Journal on Control and Optimization, vol. 42, no. 3, pp. 1071-1086, 2003.

[22] J. Jahn, Vector Optimization. Theory, Applications, and Extensions, Springer, Berlin, Germany, 2004. 


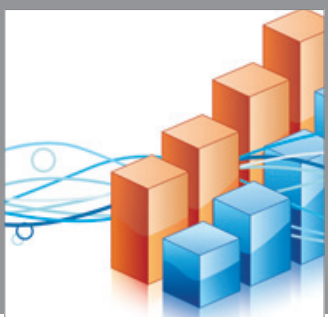

Advances in

Operations Research

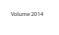

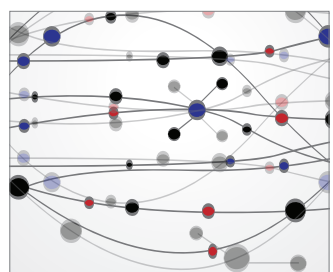

\section{The Scientific} World Journal
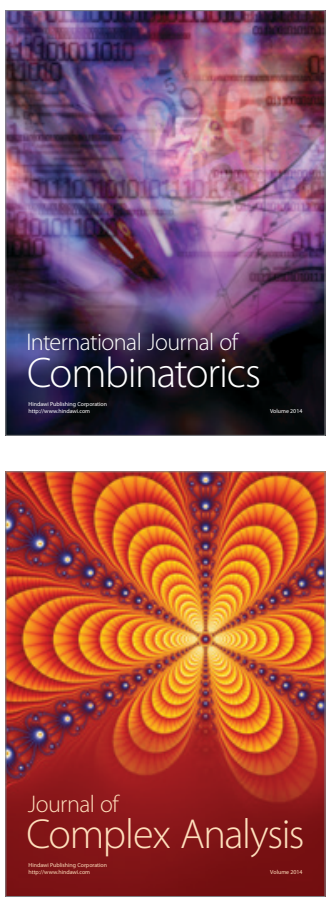

International Journal of

Mathematics and

Mathematical

Sciences
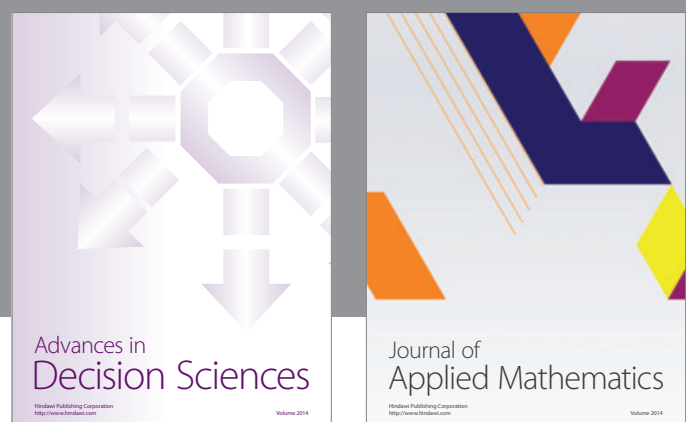

Journal of

Applied Mathematics
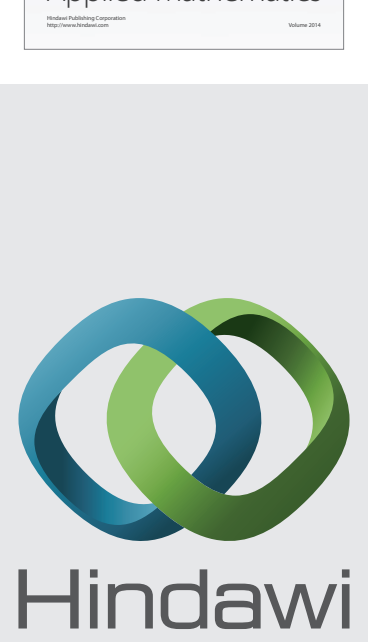

Submit your manuscripts at http://www.hindawi.com
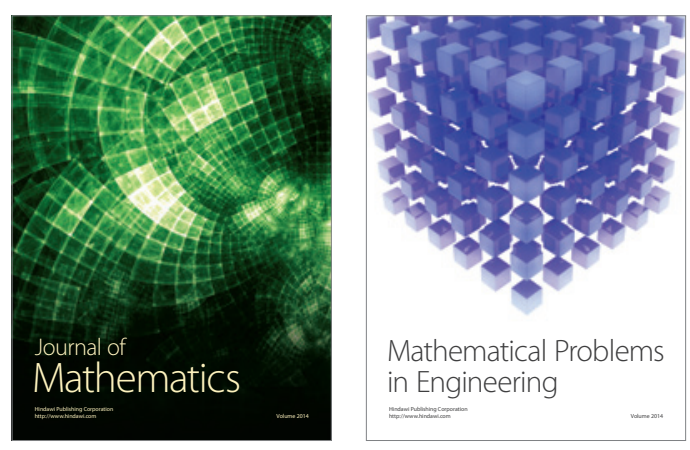

Mathematical Problems in Engineering
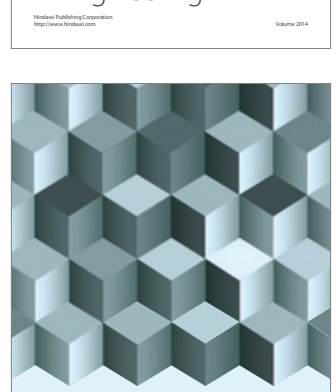

Journal of

Function Spaces
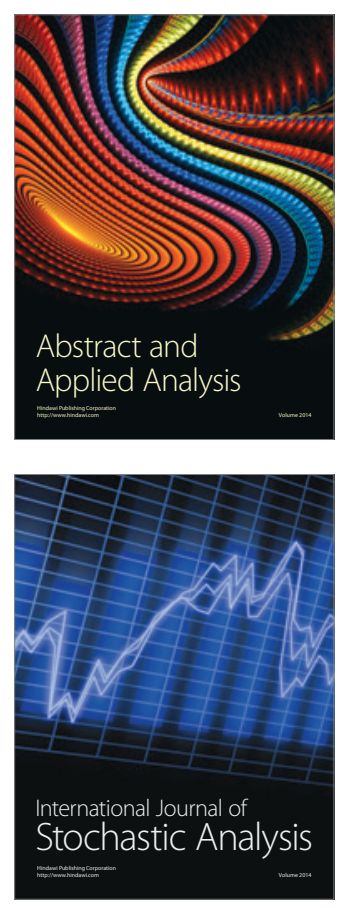

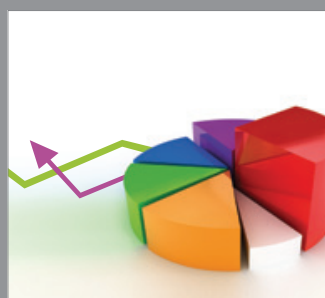

ournal of

Probability and Statistics

Promensencen
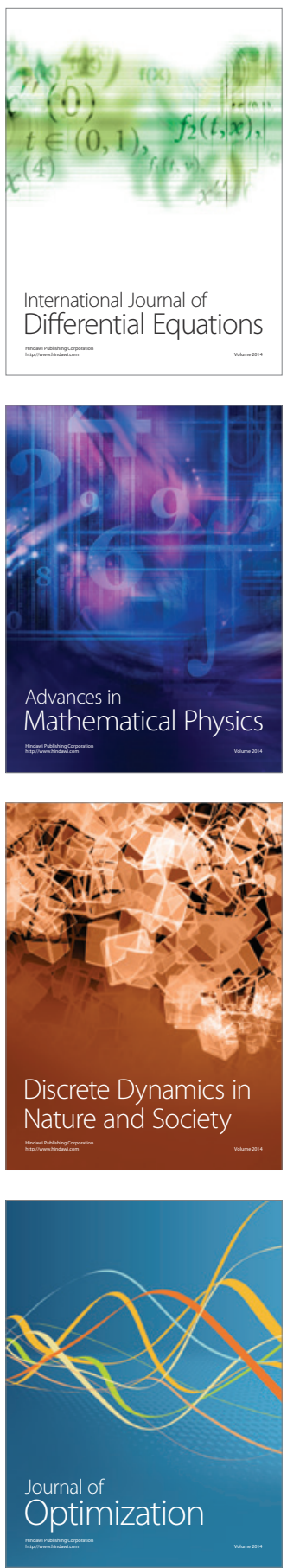